\title{
Terrain mapping camera for Chandrayaan-1
}

\author{
A S Kiran Kumar* and A Roy Chowdhury \\ Space Applications Centre, ISRO, Ahmedabad 380 015, India. \\ *e-mail: kiran@sac.isro.gov.in
}

\begin{abstract}
The Terrain Mapping Camera (TMC) on India's first satellite for lunar exploration, Chandrayaan-1, is for generating high-resolution 3-dimensional maps of the Moon. With this instrument, a complete topographic map of the Moon with $5 \mathrm{~m}$ spatial resolution and 10-bit quantization will be available for scientific studies. The TMC will image within the panchromatic spectral band of 0.4 to $0.9 \mu \mathrm{m}$ with a stereo view in the fore, nadir and aft directions of the spacecraft movement and have a $\mathrm{B} / \mathrm{H}$ ratio of 1 . The swath coverage will be $20 \mathrm{~km}$. The camera is configured for imaging in the push broom-mode with three linear detectors in the image plane. The camera will have four gain settings to cover the varying illumination conditions of the Moon. Additionally, a provision of imaging with reduced resolution, for improving Signal-to-Noise Ratio (SNR) in polar regions, which have poor illumination conditions throughout, has been made. SNR of better than 100 is expected in the $\pm 60^{\circ}$ latitude region for mature mare soil, which is one of the darkest regions on the lunar surface. This paper presents a brief description of the TMC instrument.
\end{abstract}

\section{Introduction}

The Terrain Mapping Camera (TMC) planned for India's first mission to the Moon, Chandrayaan-1, is intended for systematic topographic mapping of the complete lunar surface, including the far side and the polar regions. The aim is to prepare a 3-dimensional atlas of the Moon with a high spatial and altitude $(5 \mathrm{~m})$ resolution with 10bit quantization for scientific studies. Such a high resolution imagery of the complete Moon will be available for the first time and will help in a better understanding of the evolution process, evaluating possible water resources on permanently shadowed polar regions and in a detailed study of regions of scientific interest. The digital elevation model available from TMC along with the Lunar Laser Ranging Instrument (LLRI) on Chandrayaan-1 could improve the Moon gravity model. TMC is configured for imaging in the push broom-mode within the panchromatic spectral band of 0.4 to $0.9 \mu \mathrm{m}$. For obtaining the elevation information the camera will have an along-track stereo viewing, acquiring stereo triplet images of the target scene. This planned instrument will be compact, having a low weight and power dissipation.

\section{System specifications}

The purpose of TMC is to generate a high-resolution map of the Moon using stereoscopic camera. The $5 \mathrm{~m}$ spatial resolution of the camera is chosen to be commensurate with 1:50,000 scale mapping for scientific use and the desirable contour interval of less than $10 \mathrm{~m}$ for height information. The base to height ratio $(\mathrm{B} / \mathrm{H})$ of the camera will be 1 . The $\mathrm{B} / \mathrm{H}$ will be achieved by triplet viewing of the surface in the along-track direction of satellite in the fore $\left(+25.02^{\circ}\right)$, nadir $\left(0^{\circ}\right)$ and aft $\left(-25.02^{\circ}\right)$ directions. With this arrangement all regions can be imaged without occlusion with at least one stereo pair. The viewing geometry of the camera is shown in figure 1.

For the $100 \mathrm{~km}$ polar orbit, the equatorial shift of the Moon between two orbits is about $32.62 \mathrm{~km}$.

Keywords. Terrain mapping; active pixel sensor; digital elevation map; lunar topography.

J. Earth Syst. Sci. 114, No. 6, December 2005, pp. 717-720

(C) Printed in India. 


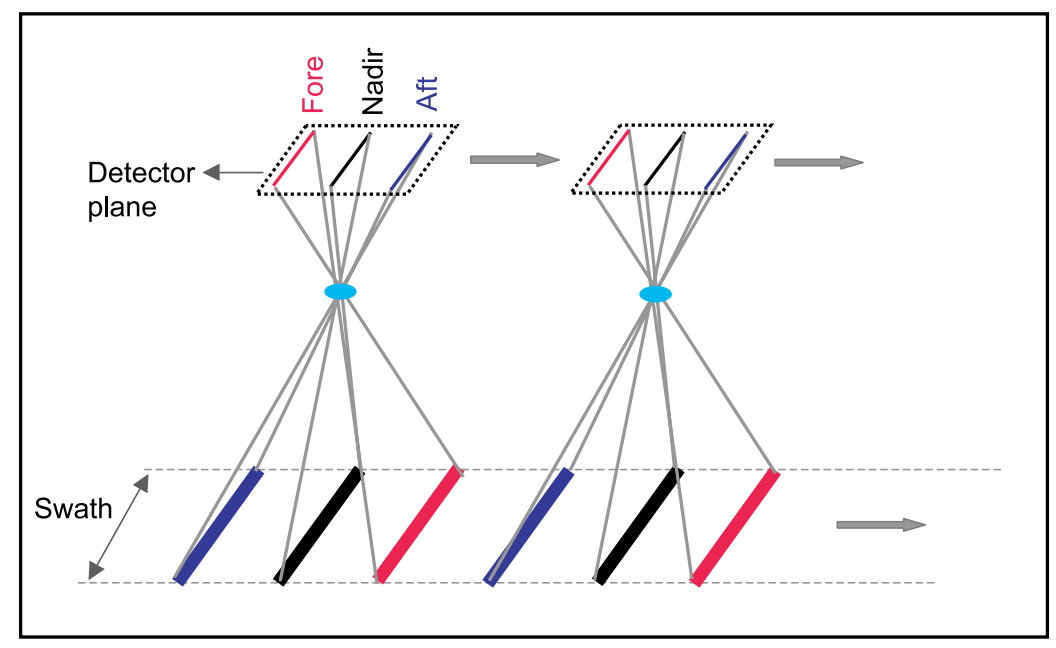

Figure 1. Viewing geometry of terrain mapping camera.

The swath is chosen as $20 \mathrm{~km}$ with orbital shift of $20 \mathrm{~km}$ between two imaging seasons. This will ensure total coverage of lunar surface at equator with adequate overlap.

Sun synchronous orbit is not planned, as this requires orbit manoeuvering. The solar illumination changes as the Moon moves in its orbit, hence the imaging time is limited to minimize the variation of illumination conditions. Limiting the solar aspect angle to $\pm 30^{\circ}$ at equator will provide two months of prime imaging time once in six months. During the prime imaging period, regions between $\pm 60^{\circ}$ latitude will be covered. This will require 20 minutes of imaging per six visible orbits from the single Indian ground station to cover the whole surface. The solar illumination at polar regions, above $60^{\circ}$ latitude, is poor in all seasons and is planned to be covered during the secondary imaging seasons.

TMC will measure the solar radiation reflected/ scattered from the Moon's surface. The dynamic range of the reflected signal is quite large $(>300)$, represented by the two extreme targets - fresh anorthosite surface and mature mare soil (Lucey et al 1994; Tompkins et al 1999; Stertel et al 2004). The other factors affecting the illumination are the seasonal variation, latitude-longitude of the scene and anisotropic reflectance of the lunar surface. The radiometric range of 1024 levels is planned to cover the total dynamic range. Four programmable gain settings are also planned. Additionally in the polar region, where illumination is poor at all times, SNR improvement will be achieved at the cost of reduced resolution, by pixel binning. Binning in the along-track direction will be achieved by setting the integration time ' $n$ ' times the dwell time. The specification of TMC is shown in table 1 .
Table 1. Specifications of terrain mapping camera.

\begin{tabular}{|c|c|}
\hline $\begin{array}{l}\text { 1. Ground and altitude re- } \\
\text { solution }(\mathrm{m})\end{array}$ & $5 \mathrm{~m}$ from $100 \mathrm{~km}$ altitude \\
\hline 2. Swath $(\mathrm{km})$ & $\sim 20$ \\
\hline $\begin{array}{l}\text { 3. Spectral band and band } \\
\text { width }(\mu \mathrm{m})\end{array}$ & $\begin{array}{l}\text { One panchromatic band } \\
\text { within } 0.4-0.9\end{array}$ \\
\hline 4. Stereo imaging & $\begin{array}{l}\text { Stereo triplet with fore, } \\
\text { nadir and aft views }\end{array}$ \\
\hline 5. No. of gains & 4 \\
\hline 6. Quantization (bits) & 10 \\
\hline $\begin{array}{c}\text { 7. SNR }\left( \pm 60^{\circ} \text { latitude for }\right. \\
\text { mature mare soil })\end{array}$ & $>100$ \\
\hline 8. Polar coverage & $\begin{array}{l}\text { SNR improvement by } \\
\text { increasing integration } \\
\text { time up to } 32 \text { times }\end{array}$ \\
\hline 9. Estimated power (W) & 13 \\
\hline 10. Estimated weight $(\mathrm{kg})$ & 6 \\
\hline
\end{tabular}

\section{Instrument description}

The design of TMC will be a compact, single optics push broom scanning system. The TMC will stereo-image the Moon's surface with three linear detectors placed in the image plane viewing the fore, nadir and aft directions in the along-track direction of satellite movement. The detector drive electronics and the processing electronics for the detector output will be housed close to the detector unit.

The optics is designed as a single unit catering to the wide field of view (FOV) requirement in the along-track direction. The incident beam from the fore $\left(+25.02^{\circ}\right)$ and aft $\left(-25.02^{\circ}\right)$ directions is directed on to the focusing optics using mirrors. This reduces the incident angle of the beams on the focusing optics, which is then realizable with spherical elements. The nadir has an unobscured view. The focusing optics is a refractive 


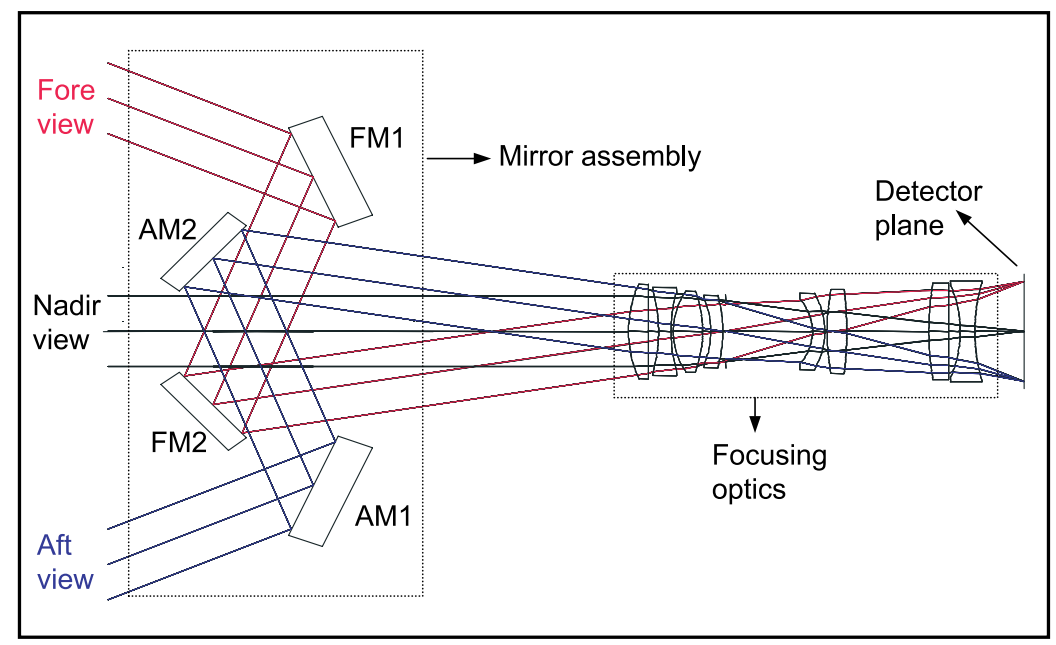

Figure 2. Optical schematic of terrain mapping camera.

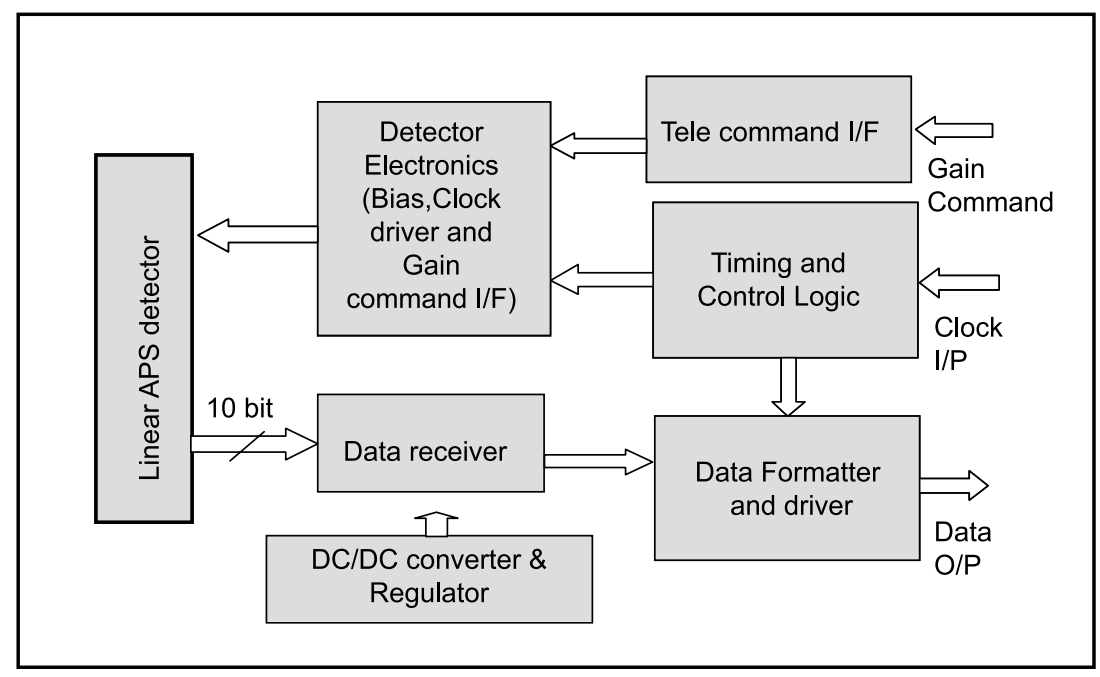

Figure 3. Block schematic of camera electronics for the detector.

eight elements $\mathrm{f} / 4$ system with FOVs of $\pm 5.7^{\circ}$ and $\pm 10^{\circ}$ in the across-track and along-track directions respectively. With this configuration, good image quality is achievable with minimum size and weight. The preliminary optical schematic is shown in figure 2.

The camera electronics will be custom designed for the detector and system requirements with the main considerations of:

- Minimization of weight, size and power.

- Electronics SNR > 1000 commensurate with 10bit digitization.

- No registration error or MTF degradation due to electronics.

It is planned to cover the complete signal range with a single gain to facilitate imaging of low and high illumination objects simultaneously. However, four programmable gains will also be provided for imaging low illumination and polar regions. The gain can be set through ground commanding. In addition, the feature of increasing scene dwell time for improving SNR will also be built in. Linear Active Pixel Sensor (APS) detector is planned considering the advantage of having the built-in detector drive and processing electronics. The output of the detector will be in digitized form. This will reduce the external hardware and will minimize the overall power, weight and size. Three detectors will be placed in the focal plane for triplet stereo viewing. The electronics is planned to be modular and separate for the three detectors. The block schematic of the camera electronics for a detector is shown in figure 3 . The preliminary power estimation for the instrument is about $13 \mathrm{~W}$.

The consideration of the mechanical housing of the instrument is to realize the performance over 


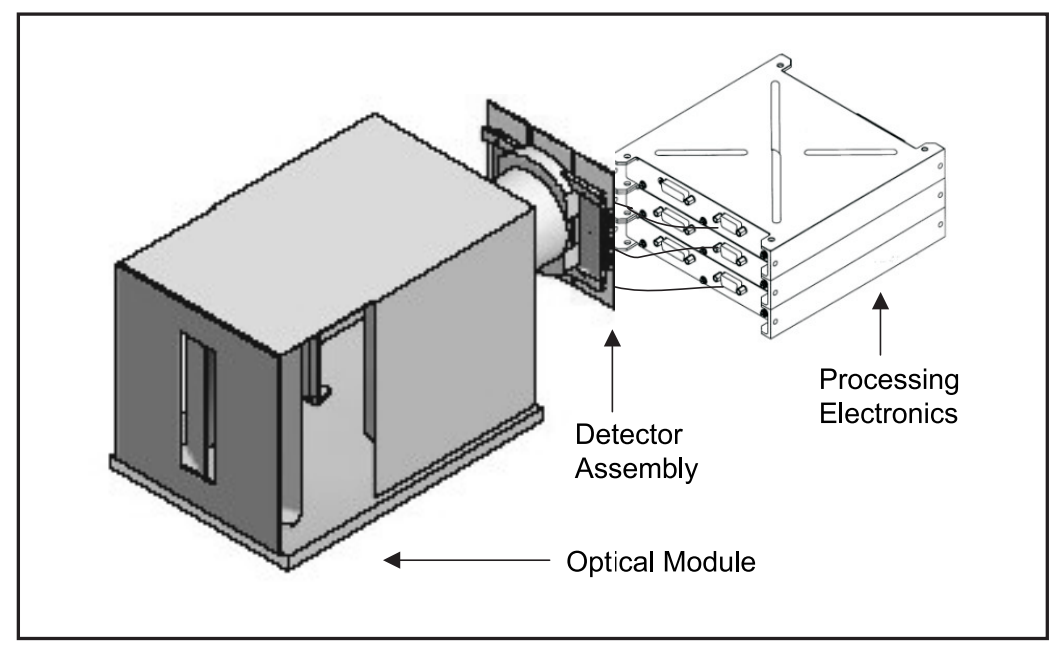

Figure 4. Schematic of terrain mapping camera instrument.

the operating temperature with minimum weight and size. The electronics will be housed separately keeping minimum interface length with the detector, for minimizing thermal effects on optics and the detector. The estimated weight of the instrument is about $6 \mathrm{~kg}$. The schematic of the instrument is shown in figure 4 .

\section{Acknowledgements}

The authors acknowledge with thanks the contributions from D R M Samudraiah, Saji Kuriakose, Chirag Dewan, Kurien Mathew, S S Sarkar, S R Joshi and V D Patel. We also gratefully acknowledge the encouragement and guidance received from Dr. K N Shankara, Director and Dr. Ram Rattan, Deputy Director, Space Applications Centre.

\section{References}

Lucey P G, Spudis P D, Zuber M, Smith D and Malaret E 1994 Topographic - Compositional Units on the Moon and the Early evolution of the lunar crust; Science $\mathbf{2 6 6}$ $1855-1858$.

Stertel D, Lucey P G and Gillis J J 2004 NearInfrared Spectral Properties of selected Nearside and Farside sites; Lunar and Planetary Science XXXV (CD Rom).

Tompkins S and Pieters C M 1999 Mineralogy of the lunar crust: Results from Clementine; Meteoritics Planet. Sci. $3425-41$. 